\title{
Comparison of Cross Fractionation and TLC Methods in the Determination of the Compositional Distribution of a Statistical Copolymer
}

\author{
Shinya Teramachi, Akira Hasegawa, and Shunichi YoshidA \\ Department of Industrial Chemistry, Kogakuin University, \\ 2665-1 Nakano-cho, Hachioji, Tokyo 192, Japan.
}

(Received October 16, 1981)

\begin{abstract}
The chemical composition distribution (CCD) of a high-conversion sample of styrene-methyl acrylate statistical copolymer was determined by thin-layer chromatography (TLC). The CCD was compared with the CCD determined by cross fractionation and also with that calculated theoretically in our previous work. The agreement among the three CCD's was sutisfactory.
\end{abstract}
KEY WORDS Chemical Composition Distribution / Thin-Layer Chromatography / Cross Fractionation / Styrene-Methyl Acrylate Copolymer / High-Conversion /

The determination of the chemical composition distribution (CCD) is important for the molecular characterization of stastistical copolymers. Among various methods proposed, a few, ${ }^{1-11}$ such as cross fractionation, ${ }^{1-6}$ thin layer chromatography (TLC) ${ }^{7.8}$ can give quantitative CCD.

Strictly speaking, however, the cross fractionation method should generally give a CCD narrower than the true one, whereas it is inevitable from the principle of chromatography that the TLC method tends to give an apparent $\mathrm{CCD}$ broader than the true one. Therefore, it is very interesting to compare the CCD's determined by both methods using a sample with a broad CCD. It is also well known that if the CCD of a sample is too sharp, both methods may give a false and broader CCD.

In the present work, the $\mathrm{CCD}$ of a highconversion sample of styrene (St)-methyl acrylate (MA) copolymer, used for the study of cross fractionation in our previous work, ${ }^{6}$ was determined by TLC method. The CCD determined by TLC was compared with the CCD determined by the cross fractionation method and also with that calculated theoretically in our previous work. ${ }^{6}$

\section{EXPERIMENTAL}

\section{Samples}

The sample chosen for this work was the same copolymer sample (B-60) as that used in previous studies. ${ }^{6,12}$ The sample was obtained by polymerizing a monomer mixture containing $60.0 \mathrm{~mol} \%$ of methyl acrylate to $92.0 \mathrm{wt} \%$ conversion in bulk. The average MA-content of the sample was $56.4 \mathrm{~mol} \%$ by elemental analysis and $56.0 \mathrm{~mol} \%$ by ${ }^{1} \mathrm{H}$ NMR. The number-average molecular weight $M_{n}$ was $4.8_{2} \times 10^{5}$.

Low-conversion samples of $\mathrm{N}-45(\mathrm{MA} / \mathrm{mol} \%=$ 46.6, $\left.M_{n} \times 10^{5}=2.6_{1}\right), \mathrm{N}-60\left(57.3,2.7_{6}\right)$, and N-75 $\left(77.9,3.0_{2}\right)$ and a homopolymer of methyl acrylate, PMA, were used as the reference samples for the TLC experiments. The preparation of these samples and the methods for their characterization are presented in our previous paper. ${ }^{12}$

\section{TLC Experiments}

The experimental procedures for TLC were similar to those described by Inagaki et al. ${ }^{7}$ Precoated plates (Analtech Uniplate Silicagel GF-2511) were used. The absorbent, silicagel, was activated by heating the plates at $110^{\circ} \mathrm{C}$ for $1 \mathrm{~h}$ before use. Polymer samples of $20 \mu \mathrm{g}$ were deposited with a 
microsyringe as spots from $2 \mu \mathrm{g} \mu \mathrm{l}^{-1}$ chloroform solutions $(10 \mu \mathrm{l})$ along a line $2 \mathrm{~cm}$ from the edge of the plate. After $10 \mathrm{~min}$ air drying, the plate was placed in a chamber containing a mixture of $\mathrm{CCl}_{4}$ and methyl acetate $1 \mathrm{~cm}$ in depth.

Gradient elution was performed by adding methyl acetate at a constant rate using a micropump. The methyl acetate content was linearly varied from 6.0 to $43.0 \mathrm{vol} \%$. When the migration distance of the solvent front reached $10 \mathrm{~cm}$, the plate was removed from the chamber and dried by a hot air-stream.

A sulfuric acid solution of $3 N$ was sprayed over the plate which was then heated at $250 \mathrm{C}$ for $1 \mathrm{~h}$, causing the positions of the polymers to become visible as dark brown spots or streaks.

The amount of polymer was determined by a Shimadzu High-speed Chromatoscanner, model CS-920 (Shimadzu Seisakusho Co., Japan). The chromatogram of blackness as a function of migration distance was obtained by a zig-zag scanning of a sample spot or streak.

\section{RESULTS AND DISCUSSION}

\section{Preliminary Tests}

Three preliminary tests are required for converting a chromatogram obtained by a scanner to a CCD. First, the linear relationship between the amount of sample spotted and the area of the chromatogram (blackness) was examined. This area was proportional to the amount of sample up to about $15 \mu \mathrm{g}$ for each reference sample $(\mathrm{N}$-series samples and PMA), provided the sample was dissolved in $10 \mu \mathrm{l}$ and spotted in an area $2.5-2.8$ mm diameter.

Second, the relationship between the chromatogram area and the MA-content of the reference samples at a constant polymer amount $(10 \mu \mathrm{g})$ was determined by measuring the chromatogram areas of 16 spots for each reference sample. The relationship obtained is shown in Figure 1.

Third, the relationship between the $R_{f}$-value and the MA-content of the sample was determined, as shown in Figure 2. In actual practice, the data of these reference samples are taken together with the sample to be examined in all experiments.

The $R_{f}$ axis of the chromatogram obtained by the scanner can be converted to the MA-content axis by using the relationship in Figure 2 . The hight

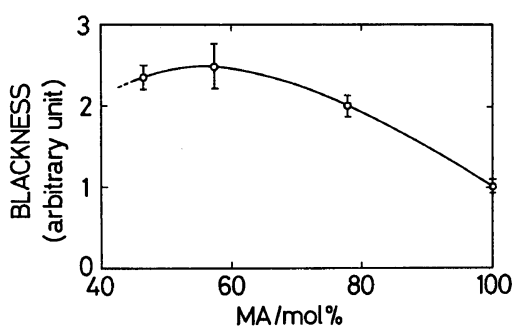

Figure 1. The relationship between chromatogram area (blackness) and MA-content of the reference samples.

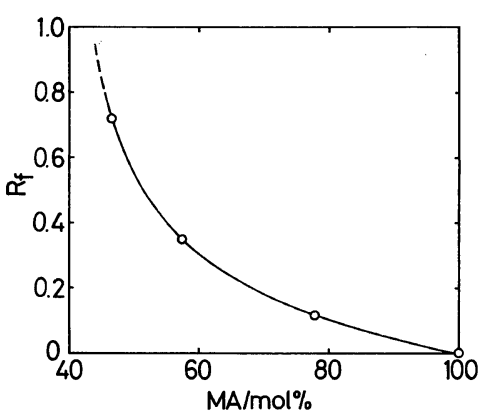

Figure 2. The relationship between $R_{f}$-value and MAcontent.

of the chromatogram at each point, $h_{i}$, was calibrated by taking into account the relative blackness at the point, $B_{i}$, in Figure 1 . The absolute value of the gradient, $\left|\Delta R_{f} / \Delta \alpha\right|_{i}$, where $\alpha$ is the $\mathrm{mol} \%$ of MA, was also obtained from Figure 2 . The relative hight, $H_{i}$, of the histogram of CCD (arbitrary unit) can thus be obtained by,

$$
H_{i}=\frac{h_{i}}{B_{i}}\left|\Delta R_{f} / \Delta \alpha\right|_{i}
$$

The integral-type CCD was obtained by normalizing $H_{i}$ and cumulating the normalized $H_{i}$.

\section{Determination of $C C D$}

The chromatograms of $\mathrm{N}-45$ and $\mathrm{B}-60$ are shown in Figure 3. Not only sample B-60 but also the Nseries samples have sub-peaks corresponding to PMA $\left(R_{f}=0\right)$. Moreover, PMA shows double peaks at $R_{f}=0$ and $R_{f}>0$, when the development is carried out by an eluent containing mthyl acetate (polar solvent) that is more than $43 \mathrm{vol} \%$. However, 


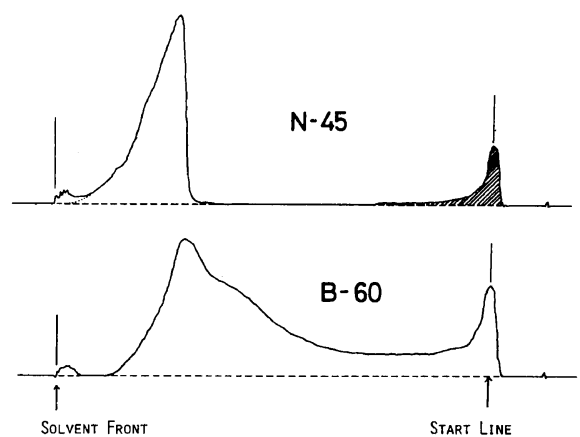

Figure 3. The TLC chromatograms of samples of $\mathrm{N}-45$ (a) and B-60 (b).

these copolymer samples should not contain PMA, as is clear from their preparation procedure. ${ }^{12}$ The CCD obtained by theoretical calculation as well as that obtained by cross fractionation did not show such a sub-peak for B-60. ${ }^{6}$ Therefore, these subpeaks at $R_{f}=0$ should be the apparent (false) ones, and may be due to a residue of sample left at the starting point as reported by Tagata and Homma for column adsorption chromatography. ${ }^{9}$

Sample N-45 is a low-conversion sample obtained from the same monomer mixture as that for B-60. The sub-peak of N-45 is clearly separated from the main peak as shown by the shaded area in Figure 3. Assuming that the apparent part at $R_{f}=0$ is equal in the chromatograms for both $\mathrm{N}-45$ and $\mathrm{B}-60$, we substracted the shaded part of $\mathrm{N}-45$ chromatogram from that of B-60 to obtain the correct CCD of B60. Thus, the integral-type CCD of both B-60 and $\mathrm{N}-45$ were calculated from the original chromatograms in Figure 3, as shown by solid (a) and dotted (b) lines in Figure 4, and the CCD of N-45 in the region of $70-100 \mathrm{~mol} \%$ of MA-content was substracted from the CCD of B-60, and the residual $\mathrm{CCD}$ of $\mathrm{B}-60$ was again renormalized.

The corrected CCD of B-60 thus obtained is also shown by a dashed line (c) in Figure 4, and is compared with the CCD's obtained from cross fractionation and the theoretical calculation in the previous work ${ }^{6}$ in Figure 5.

The agreement among the three CCD's in Figure 5 is satisfactory. The average MA-content calculated from the uncorrected CCD [Figure 4(a)] does not agree with the value for the original sample, but the value calculated from the corrected CCD

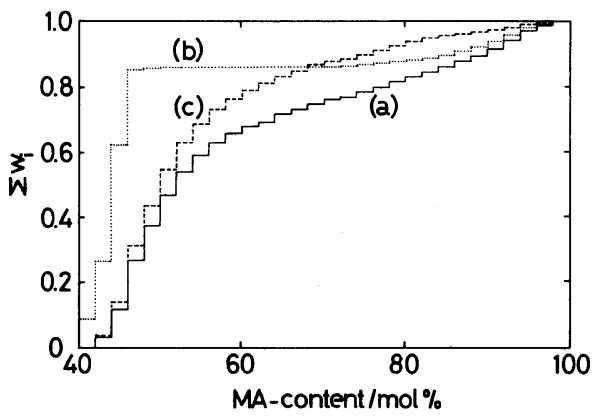

Figure 4. The CCD's obtained from TLC chromatograms: (a), uncorrected CCD of B-60, (b), uncorrected CCD of N-45; (c), corrected CCD of B-60.

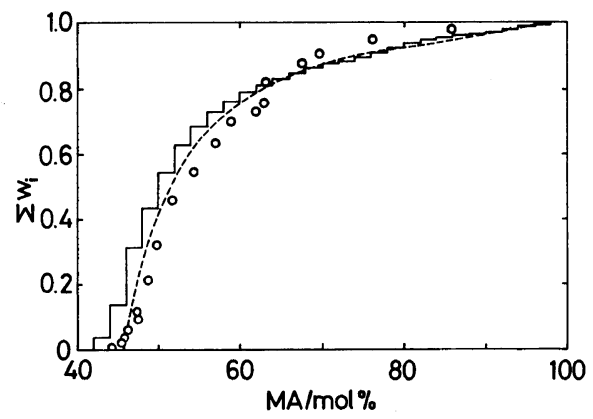

Figure 5. The CCD's of B-60 obtained by TLC method $(\ulcorner)$, cross fractionation $(O)$ and theoretical calculation (---).

Table I. The average MA-content of sample B-60

\begin{tabular}{lc}
\hline & MA \\
\cline { 2 - 2 } & $\mathrm{mol} \%$ \\
\hline Original sample & \\
Elemental analysis & 56.4 \\
${ }^{1} \mathrm{H}$ NMR method & 56.0 \\
Average from cross fractionation & 56.1 \\
Average from TLC method & \\
$\quad$ Corrected & 55.7 \\
Uncorrected & 60.4 \\
\hline
\end{tabular}

[Figure 4(c)] is in good agreement with the original value, as shown in Table I. From these agreements, we may conclude that the above procedure for correction is valid. 
The residue at the starting point was not reported by Inagaki et al., ${ }^{7}$ who used reference samples having almost the same sharp CCD as in this work. This difference in observation may be due to the difference in the activities of the adsorbents used and/or to the difference in the extent of drying of the sample spots. When CCD is determined by TLC, however, a peak is sometimes observed at the start line. In such a case, the correct CCD should be obtained by substracting its contribution. Since it is not yet clear as to whether the residue at $R_{f}=0$ is independent of the chemical composition of the sample, it is advisable to use a low-conversion sample as the reference sample to determine the apparent part.

Figure 5 also shows that the TLC method gives a broader $\mathrm{CCD}$ and the cross fractionation gives a narrower $\mathrm{CCD}$ than the theoretical one, as was predicted.

Acknowledgment. The authors gratefully acknowledge the helpful comments made by Professor Mitsuru Nagasawa of Nagoya University in carrying out this work.

\section{REFERENCES}

1. V. A. Agasandyan, L. G. Kudryavtseva, A. D. Litomanovich, and V. Ya. Shtern, Vysokomol. Soedin., Ser. A, 9, 2634 (1967).

2. S. Teramachi and Y. Kato, J. Macromol. Sci., Chem., 4, 1785 (1970).

3. S. Teramachi and Y. Kato, Macromolecuels, 4, 54 (1971).

4. S. Teramachi and T. Fukao, Polym. J., 6, 532 (1974).

5. J. Stejskal and P. Kratochvil, Macromolecules, 11, 1097 (1978).

6. S. Teramachi, A. Hasegawa, S. Hasegawa, and T. Ishibe, Polym. J., 13, 319 (1981).

7. H. Inagaki, H. Matsuda, and F. Kamiyama, Macromolecules, 1, 520 (1968).

8. Wälchli, T. Miyamoto, and H. Inagaki, Bull. Inst. Chem. Res., Kyoto Univ., 56, 80 (1978).

9. N. Tagata and T. Homma, Nippon Kagaku Kaishi, 1330 (1972).

10. T. Tanaka, M. Omoto, N. Donkai, and H. Inagaki, J. Macromol. Sci., Phys., B17, 211 (1980).

11. S. Teramachi, A. Hasegawa, Y. Shima, M. Akatsuka, and M. Nakajima, Macromolecules, 12, 992 (1979).

12. S. Teramachi, A. Hasegawa, M. Akatsuka, A. Yamashita, and N. Takemoto, Macromolecules, 11, 1206 (1978). 